\title{
Meditation, Mäuse und Myelin
}

\author{
M. Spitzer, Ulm
}

Meditation ist seit geraumer Zeit Gegenstand der Gehirnforschung (13). Beim Menschen wurden sowohl funktionelle als auch strukturelle Änderungen im Gehirn beschrieben: Zunahme der Dicke des Kortex, vermehrte oder verminderte kortikale Aktivierung in Ruhe oder bei bestimmten Aufgaben; Veränderungen der funktionellen Konnektivität; Veränderungen der strukturellen Konnektivität und der axonalen Myelinisierung und damit der Informationsübertragungsgeschwindigkeit im Gehirn.

\section{Beim Menschen wurden durch} Meditation funktionelle und strukturelle Änderungen im Gehirn beschrieben.

Insbesondere einer der Vordenker der kognitiven Neurowissenschaft, Michael Posner von der University of Oregon in Eugene, untersucht seit geraumer Zeit die Auswirkungen von standardisiert durchgeführter „Mindfulness meditation“ (hierzulande würde man wahrscheinlich am ehesten von "Achtsamkeitstraining" sprechen). Als Spezialist für Aufmerksamkeit fand er vor mehr als 10 Jahren in einer Studie an 40 Studenten, dass auch ein relativ kurzes Meditationstraining - 20 Minuten Training an 5 aufeinanderfolgenden Wochentagen - einen messbaren Effekt in entsprechenden objektiven Tests von kognitiver Kontrolle und damit auch der Emotionskontrolle zur Folge hatte, nicht jedoch auf die Wachheit (,alerting") und die selektive Aufmerksamkeit („orienting“), den beiden anderen Unterfunktionen, die im verwendeten Attention Network Task, ANT, untersucht wurden (18).

\section{Nervenheilkunde 2018; 37: 745-748}

Korrespondenzadresse

Prof. Dr. Dr. Manfred Spitzer, Universitätsklinikum Ulm Klinik für Psychiatrie und Psychotherapie III

Leimgrubenweg 12, 89075 Ulm
Nur 3 Jahre später wurden zudem durch das Magnetresonanztomografieverfahren des Diffusions-Tensor-Imaging (MRT DTI) von der gleichen Arbeitsgruppe erstmals Veränderungen der weißen Substanz, also der Nervenfasern bzw. deren Myelinisierung, durch Meditation gefunden. 45 Studenten wurden randomisiert in 2 Gruppen aufgeteilt, von denen die eine 11 Stunden (in Sitzungen von jeweils 30 Minuten über einen Monat verteilt) meditierte und die andere für die gleiche Zeit Entspannungstraining (Kontrollgruppe) durchführte (19). Die Meditation führte zu einer Steigerung der fraktionellen Anisotropie, ein $\mathrm{Ma} ß$ für die Gerichtetheit von Nervenfasern, das mit dem Ausmaß von deren Myelinisierung in Zusammenhang steht. Diese Veränderung wurde für die Fasern der anterioren Corona radiata gefunden, die den anterioren Gyrus cinguli (englisch: anterior cingulate gyrus, abgekürzt ACC) mit dem Striatum und weiteren Strukturen verbindet. Der ACC wiederum spielt eine wesentliche Rolle bei der Kontrolle von Denken und Emotionen.

Die beim Menschen durch Meditation gefunden Veränderungen der weißen Substanz passen also zu den ebenfalls beim Menschen nachgewiesenen psychologischen Änderungen durch Meditation und erlauben damit Vermutungen über deren Wirkungsmechanismus. Zu diesem Thema wurden zwischenzeitlich mehrere Sammelbände, Übersichtsarbeiten und Reviews publiziert, auf die ich den Leser verweisen möchte, der tiefer in die Materie eindringen möchte (15-20). Viele von ihnen kreisen um die Hypothese, dass Mediation d. h. das Training der bewussten (topdown) Kontrolle der Inhalte unseres Geistes - zu messbaren und für unser Wohlbefinden und unsere geistige Gesundheit positiven Veränderungen von Funktion und vor allem auch Struktur der Faserverbindungen (Axone) zwischen Nervenzellen beruht. Das klingt zunächst - nicht zuletzt auch für Neurologen und Psychiater recht ungeheuerlich und bedarf daher der
Einordnung in einen größeren neurobiologischen Zusammenhang.

Das Faktum der Neuroplastizität - also der Veränderungen des Gehirns durch dessen Gebrauch - hat sich herumgesprochen. Man denkt dabei in aller Regel jedoch an die graue Substanz, d. h. an Neuronen und Synapsen. Die weiße Substanz hingegen Nervenfasern - stellte man sich statisch vor: Verbindungskabel eben, die sich nicht ändern, sieht man einmal von Krankheiten ab. Seit über 100 Jahren ist bekannt, dass die Geschwindigkeit der Weiterleitung von Impulsen (Aktionspotenzialen) an Axonen davon abhängt, ob diese Fasern mit einer oder mehreren Schichten eines bestimmten fettigen Isolierstoffs - dem Myelin umwickelt sind. Dadurch werden diese Fasern dicker und das Größenwachstum des Gehirns nach der Geburt geht vor allem auf dieses Dickerwerden der Axone - ihre Umwicklung mit Myelinscheiden - zum Zweck der rascheren Leitung von Signalen zurück. Man stellte sich diese Myelinisierung der Nervenfasern als ganz normalen Wachstumsprozess vor, etwa so, wie der Knochen wächst oder die Haare.

Man stellte sich diese Myelinisierung der Nervenfasern als ganz normalen Wachstumsprozess vor.

Noch vor 25 Jahren war die Sache mit der Myelinisierung von Faserverbindungen zwischen Axonen einfach: Wir werden mit weitgehend unmyelinisierten Axonen geboren (im Wesentlichen sind lediglich primär sensorische und motorische Fasern zum Zeitpunkt der Geburt mit Myelinscheiden ,umwickelt") und dann geschieht die Myelinisierung von Axonen im gesamten Gehirn im Verlauf von dessen Entwicklung im ersten Lebensjahrzehnt. Sie folgt dabei einem bestimmten Schema - funktional „höhere" Gehirnzentren (Denken, Planen, Wollen, Bewerten) kommen später dran als ,einfachere“ (Sensorik, Motorik) und läuft einfach $a b$. 
Herausgefunden hatte dies der bekannte Anatom Paul Flechsig (5) bereits vor etwa 100 Jahren, indem er Gehirnschnitte von Menschen verschiedenen Sterbealters mit einem Farbstoff einfärbte, der Fett schwarz färbt ( $\downarrow$ Abb 1). „Das Gehirn ist bei der Geburt eines Menschen noch nicht voll entwickelt. Beim Neugeborenen wiegt es etwa 350 Gramm, beim Erwachsenen hingegen 1300 (Frau) bis 1400 (Mann) Gramm. Das Gehirn des Neugeborenen hat also nur etwa ein Viertel des Gewichts und der Größe des Gehirns eines erwachsenen Menschen, obwohl sowohl die Neuronen als auch deren Verbindungsfasern bereits vorhanden sind und nach der Geburt zahlenmäßig kaum zunehmen. Dennoch entwickelt sich das Gehirn nach der Geburt noch deutlich weiter und vervierfacht sein Gewicht. Es ist vor allem Fett, das im Laufe der Entwicklung nach der Geburt das Gehirn so groß werden lässt" fasste ich diese Überlegungen von Flechsig schon vor einem Jahrzehnt an dieser Stelle kurz zusammen (14). Von dort stammt auch die Abbildung, die mich heute nicht weniger begeistert als damals ( Abb. 1).

Myelinscheiden, durch welche die Axone mit Fett umwickelt werden, bewirken dass Aktionspotenziale nicht mehr das Axon entlang „laufen“, sondern von einem kleinen Einschnitt in der Isolierung, dem Ranvierschen Schnürring (muss man sich ihn wie die Abbindungen einer ganzen Girlande von Würstchen vorstellen; $>A b b .2$ ), zum nächsten Ranvierschem Schnürring springen. Durch diese saltatorische Erregungsleitung (lateinisch: saltus: Sprung) steigt die Geschwindigkeit der Impulse auf das bis zu 35-Fach an, von 1-3 m/s (je nach Dicke des Axons; dicke „Kabel“ leiten schneller) auf bis zu $105 \mathrm{~m} / \mathrm{s}$.

Sozusagen im Schlepptau der Erkenntnisse zur Neuroplastizität der "grauen Substanz" - von mikroskopischen Synapsenebene (Synapsen ändern ihre Stärke mit ihrer Benutzung) bis zur makroskopischen Ebene der kortikalen Karten (was dort repräsentiert ist, ändert sich mit deren Benutzung) - wurden in zunehmendem Maße auch aktivitätsabhängige Veränderungen der weißen Substanz gefunden: Die Myelinisierung von Axonen ändert sich auch in Abhängigkeit von deren Nutzung, d. h. davon, wie viele Signale über sie lau-

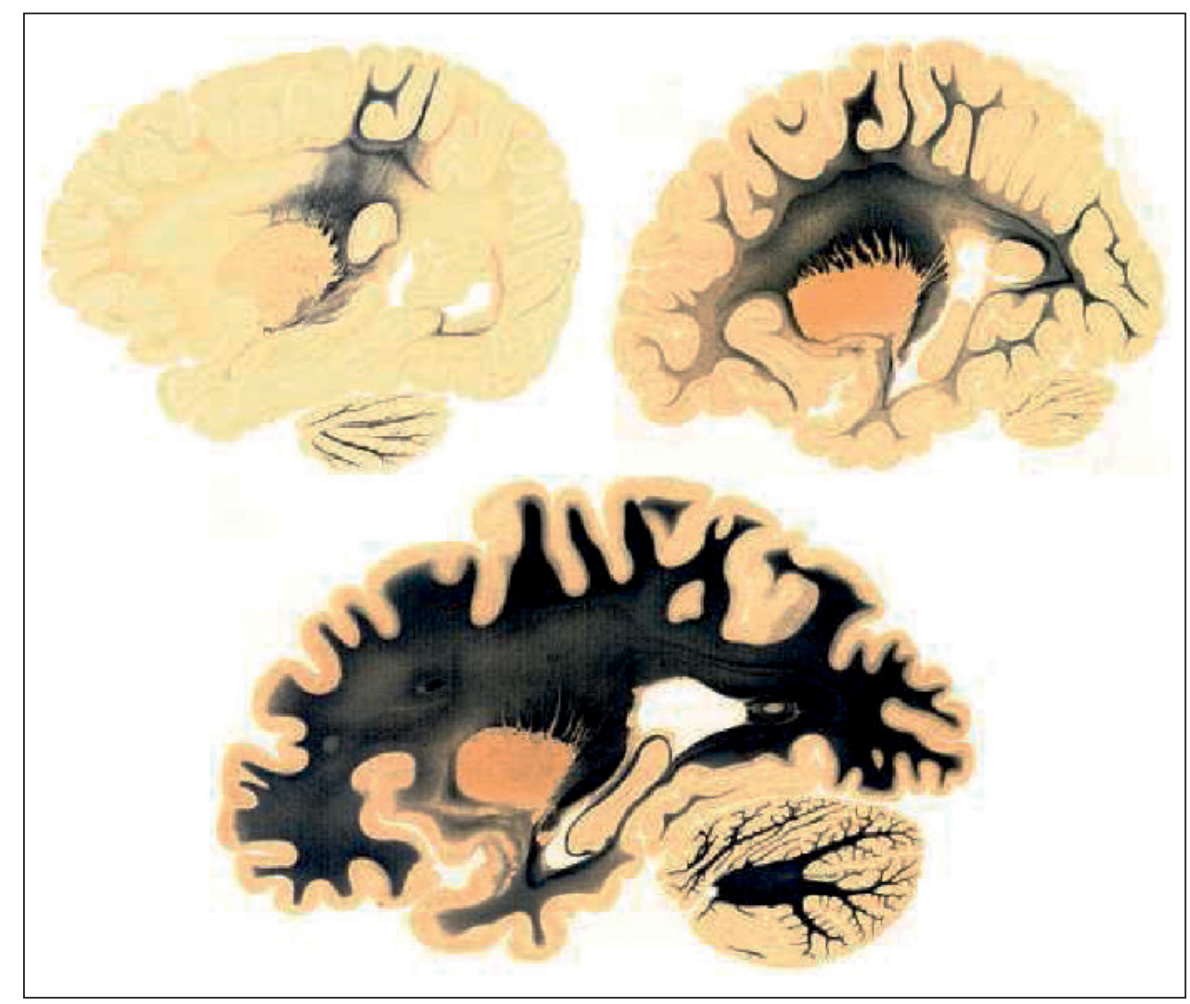

Abb. 1 Myelinisierung (Darstellung durch Anfärbung von Fett mit einer schwarzen Farbe) der Faserverbindungen kortikaler Areale. Links oben im Bild ein Schnitt durch das Gehirn eines Neugeborenen, rechts der Gehirnschnitt von einem Kind im Kindergartenalter und unten ist der Schnitt durch das Gehirn eines Erwachsenen dargestellt. Zum Zeitpunkt der Geburt sind lediglich die primären sensorischen und motorischen Areale myelinisiert, also Hirnrindenbezirke, die für die primäre Verarbeitung von Sehen, Hören und Tasten verantwortlich sind sowie zum Ausführen von Bewegungen gebraucht werden. Damit kann der Säugling erste Erfahrungen machen und reflexhaft reagieren, die Information jedoch noch nicht sehr tief verarbeiten. Später werden sekundäre und noch später tertiäre, supramodale Areale, also vor allem Frontal- und Parietalhirn, mit myelinisierten und damit schnell leitenden Fasern verbunden (nach 5, reref).

fen. So änderte sich unser Verständnis der für die Bildung der Myelinscheiden zuständigen Oligodendrozyten und deren Vorläuferzellen radikal: Aus „passivem Isoliermaterial" wurde ein plastischer aktiver Gestalter der - aktivitätsabhängigen - Gehirnfunktion! Nicht nur die Schaltelemente (Neuronen, graue Substanz) sind plastisch; die Verbindungskabel zwischen ihnen sind es auch. Die Telekom würde das so ausdrücken: Um das System zu optimieren, wird die Bandbreite der Informationsübertragung zwischen einzelnen Komponenten des Netzes den Bedürfnissen permanent angepasst. Nur so holt man bei vorgegebener Größe (Gesamtmaterialeinsatz) das Optimum aus dem Netz heraus.

Nachdem im Jahr 1993 von den britischen Neurobiologen Ben Barres und Martin Raff im Fachblatt Nature Studien am Sehnerv der Ratte publiziert worden wa- ren, die gezeigt hatten, dass eine Verminderung der Anzahl der von ihm weitergeleiteten Aktionspotenzial zu einer Verringerung des Nachwachsens der ihn isolierenden Oligodendrozyten führt, ging der Erkenntnisfortschritt erst so richtig los: Der wichtigste Neurotransmitter erregender Synapsen im Gehirn von Säugetieren ist Glutamat. Entsprechend wurden glutamaterge Synapsen auf den Vorläuferzellen von Oligodendrozyten gefunden, über den Signalverkehr in den Axonen, die sie isolieren, informiert werden, zunächst im Hippocampus (4) und ein paar Jahre später generell in der weißen Substanz (9). Dieses Signal kommt vor allem von noch nicht „umwickelten" Axonen und sorgt damit für deren Myelinisierung (22). So werden mittelfristig diejenigen Fasern aktiv zur Isolierung ausgewählt, die es am meisten nötig haben (8), bis auf die Anzahl der „Umwick- 


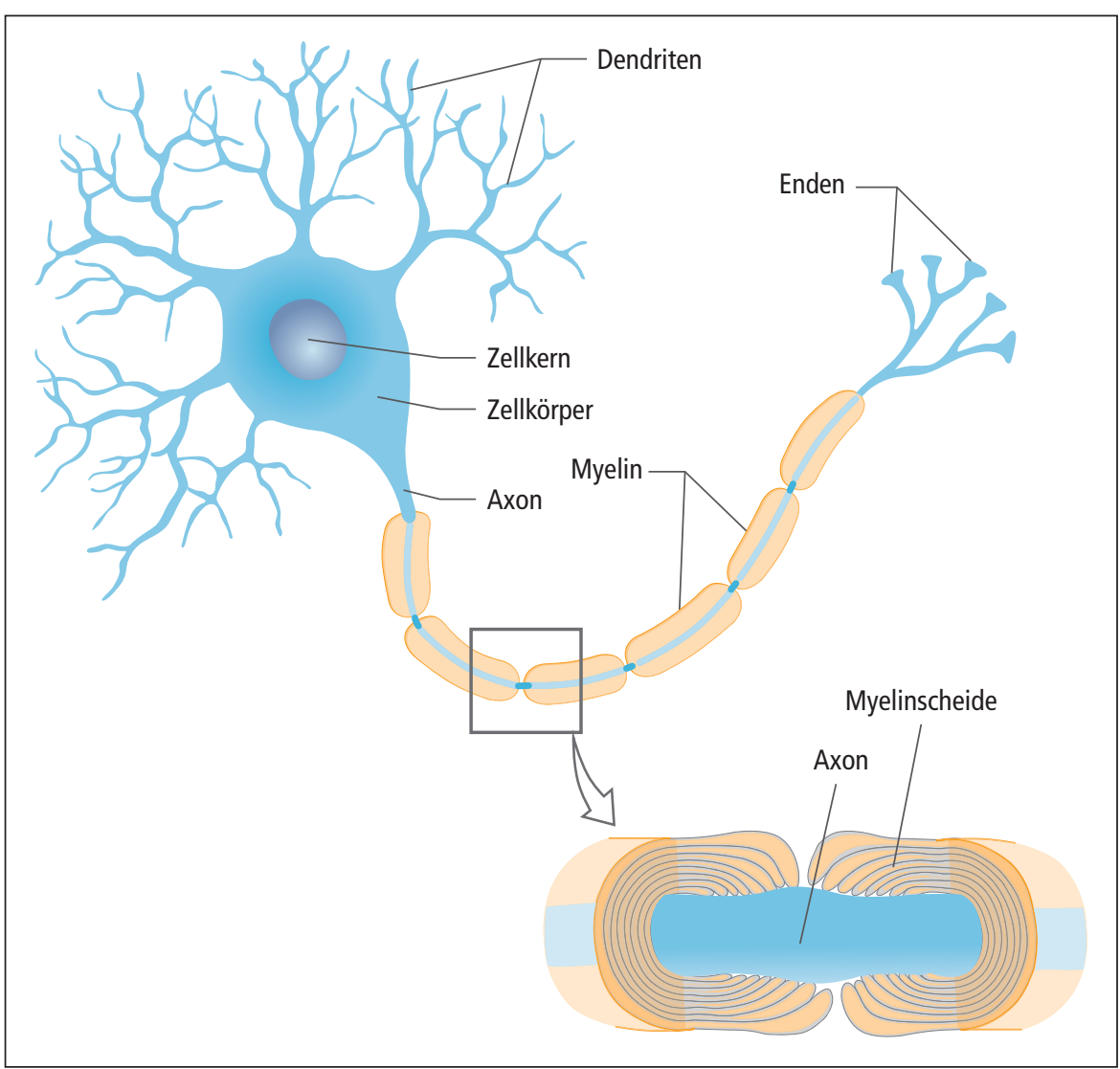

Abb. 2 Schematische Darstellung eines Neurons mit ummanteltem Axon und Einschnürungen. Oligodendrozyten, eine Unterform der Gliazellen, bewirken die Myelinisierung.

lungen" eines Axons durch einzelne Oligodendrozyten (10).

Das Gesamtsystem ist genial: „Neuronal activity promotes oligodendrogenesis and adaptive myelination in the mammalian brain" lautet der Titel einer im Jahr 2014 hierzu im Fachblatt Science publizierten Arbeit, die dies bei Mäusen in vivo mittels optogenetischer Verfahren klar zeigen konnte (7). „Die axonale Freisetzung von Glutamat erfolgt reliabel und liefert ein stark lokalisiertes Signal [...]. Diese axonale Neurotransmitterfreisetzung stellt einen weit verbreiteten Mechanismus dar, der eine genaue, aktivitätsabhängige Signalübertragung an der Schnittstelle von Axon und Glia in der weißen Substanz gewährleistet $^{\text {" }}$, formulieren Kukley und Mitarbeiter (9, S. 311) in ihrer Arbeit.

1 Im Original: „Axonal release of glutamate is reliable, involves highly localized [...] signaling [...]. This axonal transmitter release represents a widespread mechanism for high-fidelity, activity-dependent signaling at the axon-glia interface in white matter.
Zurück zur Meditation. Dieser Zustand wurde traditionell schon sehr lange dadurch untersucht, dass man Gehirnströme (EEG) ableitete und auf das Vorhandensein bestimmter Frequenzen hin analysierte. Frequenzbänder (also Bereiche bestimmter Frequenzen) wurden mit griechischen Buchstaben benannt und - ähnlich wie man beim Radio von den Frequenzbändern „Langwelle“, „Mittelwelle“, „Kurzwelle" und Ultrakurzwelle" zu sprechen gewöhnt war, sprach man von „Delta-“, „Theta-“, „Alpha-“ „Beta-“ oder „Gamma-“ Wellen im EEG.

Bereits im Jahr 2014 war deutlich geworden, dass Mediation vor allem durch Theta-Wellen im EEG im Bereich des Frontalhirns charakterisiert ist. Es wurde daher und aufgrund weiterer Indizien vorgeschlagen, dass diese Theta-Aktivität mit der gebrauchsabhängigen Myelinisierung frontaler Areale (ACC!) in Zusammenhang steht (12). Dass sie dort generiert wird, war nach den vorliegenden Indizien nicht unwahrscheinlich (21).
Wenn nun Oligodendrozyten (bzw. deren Vorläuferzellen) Informationen zum Informationsfluss in den von ihnen ummantelten Axonen bekommen und dann die Bandbreite des Netzes dem Grad der Nutzung adaptiv anpassen, wenn Meditation die Top-down-Kontrolle durch eine der obersten Kontrollinstanzen des Gehirns, den ACC, verstärkt, und wenn ThetaRhythmen an diesem Mechanismus kausal beteiligt sind, könnte man - ja was denn?

An dieser Stelle kommen noch Untersuchungen an optogenetisch manipulierten Mäusen ins Spiel (7). Diese Technik macht sich die Tatsache zunutze, dass man ein Gen aus grünen Algen, das Channelrhodopsin-2 in Neuronen von Mäusen einschleust, was zur Folge hat, dass die Zellen (zunächst wurde dies in Zellkulturen gezeigt und später auch in vivo) immer dann feuern, wenn ein grüner Lichtblitz auf sie einwirkt (1). Die Stimulation mit Licht vermeidet einige Nachteile anderer Verfahren: Elektrische Stimulation mit eingebrachten Elektroden führt immer auch zu Gewebeschäden und eingebrachter erregender Neurotransmitter (Glutamat) ist räumlich nie punktgenau und zeitlich schon gar nicht millisekundengenau (wie dies ein elektrischer Impuls oder ein Lichtblitz sein kann). Die optogenetische Stimulation gehört aus diesen Gründen seit etwa 10 Jahren zum seht gut etablierten Arsenal der Neurobiologie.

So konnte in der erwähnten, im Fachblatt Science publizierten Arbeit klar gezeigt werden, dass die neuronale Aktivität von Pyramidenzellen in Schicht 5 des Kortex (der Output-Schicht) zu einer vermehrten Genese von Oligodendrozyten und dadurch zu einer vermehrten Myelinisierung der Fasern dieser Neuronen führt, was wiederum zu einer nachweisbaren Funktionsverbesserung des Systems führte (7).

Wenn dies alles so ist, so der Gedanke von Posner und Mitarbeitern, dann müsste es doch auch möglich sein, Mittellinienstrukturen (der ACC gehört dazu) im Mausgehirn optogenetisch im ThetaRhythmus zu stimulieren, um damit den Zusammenhang derartig rhythmischer Aktivierung (wie sie beim meditierenden Menschen nachgewiesen ist) und Myelinisierung kausal nachweisen zu können. Und genau das kam auch heraus: „These 
results suggest that low-frequency stimulation can result in activity-dependent remodeling of myelin, which could lead to enhanced connectivity and altered behavior" $(11$, S. e4).

Was bedeutet dies? Zunächst einmal ist der Befund schlicht bemerkenswert! Oder hätte irgendjemand unter den Lesern dieser Zeilen vor 10 oder 15 Jahren vermutet, dass man die Grundlagen der Wirksamkeit von Meditation einmal im Mausmodell würde herausfinden können? Posner und Mitarbeiter gehen noch weiter. Es gibt ja Menschen, die Mühe mit dem Meditieren haben. Man müsste es bei denen ähnlich machen wie bei den Mäusen, nur eben ohne genetische Modifikation und Lichtleiterkabel im Gehirn. Man könnte versuchen, bei diesen Personen den ACC auf andere Weise zu stimulieren, vielleicht durch bestimmte Aufgaben oder durch nicht invasive Stimulation von außen. Lassen wir abschließend die Autoren selbst spekulieren: „Based on the findings in mice, we speculate that inducing lowfrequency rhythmic activity in humans over multiple sessions by ACC stimulation might be sufficient to alter connectivity and perhaps produce similar white matter changes found with meditation. Inducing such a rhythm less invasively in humans, whether by electrical stimulation, sensory stimulation, or neurofeedback, might work to change white matter in different brain areas and thus serve to improve functionality. The effectiveness of such stimulation might also be enhanced if accompanied by a task activating the same brain areas. If true, it might be possible to improve or restore white matter in any area of the brain for which appropriate electrodes and tasks could be designed. These ideas are preliminary, but they support the need for more research to understand the effects of brain stimulation on behavior and the underlying biological mechanisms" (11, S. e7).

\section{Literatur}

1. Arenkiel BR, Peca J, Davison IG, Feliciano C, Deisseroth K, Augustine GJ, Ehlers MD, Feng G. In vivo light-induced activation of neural circuitry in transgenic mice expressing channelrhodopsin-2. Neuron 2007; 54: 205-218.

2. Barres BA, Raff MC. Proliferation of oligodendrocyte precursor cells depends on electrical activity in axons. Nature 1993; 361: 258-260.

3. Barres BA, Raff MC. Axonal control of oligodendrocyte development. The Journal of Cell Biology 1999; 147: 1123-1128.

4. Bergles DED, Roberts JDJ, Somogyi PP, Jahr CEC. Glutamatergic synapses on oligodendrocyte precursor cells in the hippocampus. Nature 2000; 405: 187-191.

5. Flechsig P. Anatomie des menschlichen Gehirns und Rückenmarks auf myelogenetischer Grundlage. Leipzig: Thieme 1920.

6. Forbes TA, Gallo V. All Wrapped Up: Environmental Effects on Myelination. Trends Neurosci 2017; 40: 572-587.

7. Gibson EM, Purger D, Mount CW, Goldstein AK Lin GL, Wood LS, Inema I, Miller SE, Bieri G, Zuchero JB, Barres BA, Woo PJ, Vogel H, Monje $M$. Neuronal activity promotes oligodendrogenesis and adaptive myelination in the mammalian brain. Science 2014; 344: 487; 1252304 (1-12).

8. Hines J, Ravanelli A, Schwindt R, Scott E, Appel B. Neuronal activity biases axon selection for myelination in vivo. Nature Neurosci 2015; 18: 683-689.
9. Kukley M, Cepetillo-Zarate E, Dietrich D. Vesicular glutamate release from axons in white matter. Nature Neurosci 2007; 10: 311-320.

10. Mensch S, Baraban M, Almeida R, Czopka T, Ausborn J, El Manira A, Lyons DA. Synaptic vesicle release regulates myelin sheath number of individual oligodendrocytes in vivo. Nat Neurosci 2015; 18: 628-630.

11. Piscopo DM, Weible AP, Rothbart MK, Posner MI, Niell CM (2018) Changes in white matter in mice resulting from low-frequency brain stimulation. PNAS 2018; www.pnas.org/cgi/doi/10.1073/ pnas. 1802160115 .

12. Posner MI, Tang YY, Lynch G. Mechanisms of white matter change induced by meditation training. Frontiers in Psychology 2014; 5: 1220.

13. Spitzer M. Meditieren im Kopf. Nervenheilkunde 2007; 26: 1079-1082.

14. Spitzer M. Pubertät im Kopf. Nervenheilkunde 2008; 27: 674-678.

15. Tang Y-Y, Hölzel BK, Posner MI. The neuroscience of mindfulness meditation. Nat Rev Neurosci 2015; 16: 213-25.

16. Tang Y-Y, Hölzel BK, Posner MI. Traits and states in mindfulness meditation. Nat Rev Neurosci 2016; 17: 59.

17. Tang Y-Y, Lu Q, Geng X, Stein EA, Yang Y, Posner MI. Short-term meditation induces white matter changes in the anterior cingulate. PNAS 2010; 107: 15649-15652.

18. Tang Y-Y, Ma Y, Wang J, Fan Y, Feng S, Lu Q, Yu Q, Sui D, Rothbart MK, Fan M, Posner MI. Short term meditation training improves attention and self regulation. PNAS 2007; 104: 17152-17156.

19. Tang Y-Y, Posner MI. Special issue on mindfulness neuroscience. SCAN 2013; 8: 1-3.

20. Tang Y-Y, Posner MI. Tools of the trade: theory and method in mindfulness neuroscience. SCAN 2013; 8: 118-120.

21. Xue SW, Tang YY, Tang R, Posner MI. Short-term meditation induces changes in brain resting EEG theta networks. Brain Cogn 2014; 87: 1-6.

22. Ziskin JL, Nishiyama A, Rubio M, Fukaya M, Bergles DE. Vesicular release of glutamate from unmyelinated axons in white matter. Nature Neurosci 2007; 10: 321-330. 